\title{
LARGE AREA BOLOMETERS FOR MILLIMETER-WAVE POWER CALIBRATION
}

\author{
Gabriel M. Rebeiz, ${ }^{1}$ Curtis C. Ling, ${ }^{1}$ and David B. Rutledge ${ }^{2}$ \\ 'Electrical Engineering and Computer Science Department \\ The University of Michigan, Ann Arbor, Michigan 48109 \\ ${ }^{2}$ Division of Engineering and Applied Science \\ California Institute of Technology, Pasadena, California 91125
}

Received May 27, 1989

\begin{abstract}
An accurate monolithic power meter has been developed for millimeter-wave applications. The detector is a large-area Bismuth bolometer, integrated on a fused-Quartz substrate. It simply measures the temperature change caused by the absorption of millimeter-wave radiation. The power meter is simple to fabricate, inexpensive, and can be easily calibrated using a low-frequency network. The measured responsivity for a $50 \Omega$ bolometer, with an area of $1 \times 1 \mathrm{~cm}$, at a bias of $1 \mathrm{~V}$. and a video modulation of $100 \mathrm{~Hz}$, is $1 \mathrm{mV} / \mathrm{W}$. The noise spectrum exhibits a $1 / \mathrm{f}$ rolloff till $1 \mathrm{KHz}$, and is limited by the Johnson noise for higher frequencies. The NEP of the detector is $3 \mu \mathrm{W} \mathrm{Hz}^{-1 / 2}$ at a video modulation of $1 \mathrm{KHz}$. It is possible to decrease the current NEP by fabricating bolometers with higher responsivities. Possible application areas are $a b-$ solute power calibration and localized power density measurements at millimeter and submillimeter wavelengths.
\end{abstract}

\section{INTRODUCTION}

Power measurements at millimeter-wave frequencies is conventionally done using waveguide power meters. These employ a thermistor or a diode suspended in a waveguide, and become very expensive at millimeter-wave frequencies. Also, they are inaccurate for frequencies around $200 \mathrm{GHz}$, and simply not available for frequencies higher than 300GHz. Neikirk and Rutledge [1] developed a Bismuth microbolometer for millimeter-wave and far-infrared detection. The microbolometer is very small compared to wavelength, and needs a millimeter- 
wave antenna to gather the incident energy. This detector is not suited for absolute power measurements, because one must deimbed the antenna gain and mismatch from the results. Bloch and Gaddy [2] integrated a $0.75 \mathrm{~mm}$ square Bismuth bolometer for infrared-radiation detection. The measured responsivities were low because the bolometer was optimized for high-speed operation.

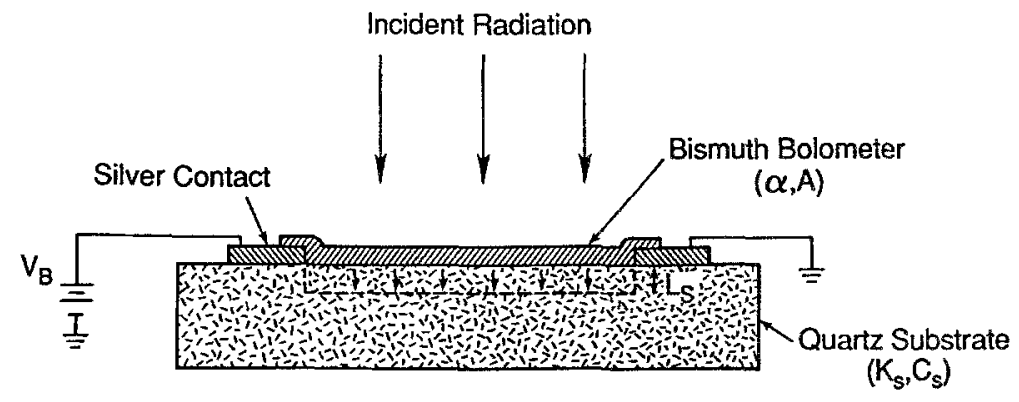

Figure 1. A monolithic large-area Bismuth bolometer.

We have developed a large-area bolometer optimized for absolute millimeter-wave power measurements (Fig.l). The bolometer is much thinner than a wavelength, and therefore presents a millimeter-wave surface impedance equal to its DC resistance per unit square. This fact, in conjunction with the bolometer large size compared to a wavelength, allows the use of a transmission line equivalent circuit to determine accurately the mismatch between a bolometer and an incident plane wave (Fig. 2). The mismatch gives the fraction of the incident power absorbed by the bolometer. If the bolometer responsivity is known using, for example, a LF calibration network, an accurate power-density measurement is then made at millimeter wavelengths. Since the bolometer is a thermal detector, it works well throughout the millimeter and submillimeter-wave regions. One only needs to change the transmission-line model to account for the frequency change.

\section{THERMAL MODEL}

The bolometer is a heat sensitive device, and will respond to the average power of an amplitude modulated signal. The bolometer heats up and cools down at the modulation frequency. This generates a heat wave that propagates a distance $\mathrm{L}_{\mathrm{S}}$ (thermal diffusion length) into the substrate. The bolometer dimensions are much larger than $\mathrm{L}_{\mathrm{S}}$, and a one dimensional heat flow model is assumed in the analysis. Neglecting 
the thermal conductance of air and the silver contacts, $\mathrm{L}_{\mathrm{S}}$ is given by [3]

$$
\mathrm{L}_{\mathrm{S}}=\sqrt{\mathrm{K}_{\mathrm{S}} /\left(2 \pi f \mathrm{C}_{\mathrm{S}}\right)}
$$

where $\mathrm{K}_{\mathrm{S}}$ and $\mathrm{C}_{\mathrm{S}}$ are the thermal conductivity and capacity of the substrate, respectively, and $f$ is the modulation frequency. The thermal diffusion length is very short in a fused-Quartz substrate, being less than $100 \mu \mathrm{m}$ for a modulation frequency of $10 \mathrm{~Hz}$. This means that, for all practical purposes, the heat wave never reaches the back of the substrate. The bolometer responsivity is the given by [3]

$$
\operatorname{Resp}=\alpha V_{b} L_{S} /\left(A_{S}\right)
$$

where $\alpha$ is the temperature coefficient of resistivity of Bismuth, $A$ is the area of the bolometer, and $V_{b}$ is the bias voltage. For a fused-Quartz substrate with $\mathrm{K}_{\mathrm{S}}=1.6 \mathrm{~W} \mathrm{~m}^{-1} \mathrm{~K}^{-1}$ and $\mathrm{C}_{\mathrm{S}}=2.36 \times 10^{6} \mathrm{Jm}^{-3} \mathrm{~K}^{-1}$, and with $\alpha=0.003 \mathrm{~K}^{-1}$ for a $1000 \AA$ Bismuth layer, the theoretical responsivity for a bolometer with the same specifications given above, is equal to $0.65 \mathrm{mV} / \mathrm{W}$.
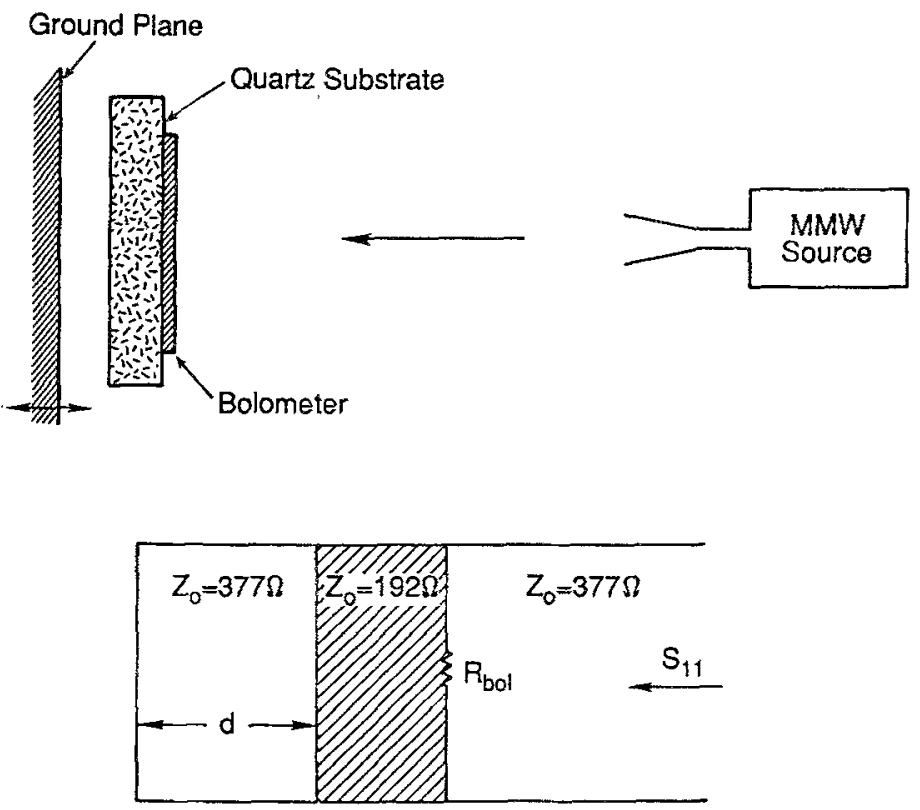

Figure 2. Millimeter-wave measurement setup, and the corresponding transmission-line equivalent circuit. 


\section{MEASUREMENTS}

The large-area bolometers are fabricated on $600 \mu \mathrm{m}$ thick fused-Quartz substrate using standard photolithography and liftoff. The silver contacts are $1000 \AA$ thick, and the bolometer is evaporated bismuth $1000 \AA-1500 \AA$ thick. The dc resistances were between $35 \Omega$ and $75 \Omega$. The frequency response and responsivity of the bolometer is measured using a low-frequency network. An amplitude modulated $4.3 \mathrm{MHz}$ signal is applied to the bolometer. The frequency response is found by varying the modulation frequency from $5 \mathrm{~Hz}$ to $10 \mathrm{KHz}$, and measuring the bolometer response using a lock-in amplifier. The frequency response can also be found using the millimeter-wave system shown in Fig. 2. In this case, an $80.7 \mathrm{GHz}$ Gunn source is modulated from $10 \mathrm{~Hz}$ to $1 \mathrm{KHz}$, and the bolometer response is measured. The output signal drops as $\mathrm{f}^{-1 / 2}$ in the LF and $80.7 \mathrm{GHz}$ set-ups, which is in agreement with the theoretical model. The output was also a linear function of the applied RF power at $80.7 \mathrm{GHz}$, and of the bias voltage.

The transmission-line equivalent circuit is checked by measuring the bolometer response at $93 \mathrm{GHz}$ with a sliding ground plane behind the Quartz substrate. The power absorbed by the bolometer is given by $\left(1-S_{11}{ }^{2}\right)$, where $S_{11}$ is calculated using the TL equivalent circuit (Fig. 2). The measurements agree well with the TL model, being always within $10 \%$ of the predicted results (Fig. 3). The discrepancies arise from standing waves between the bolometer and the source. The peaks in the measured data were higher and sharper, and were tamed by placing absorber around the bolometer.

In order to calibrate the bolometer at low-frequencies, the RF power absorbed must be accurately measured. This is done by monitoring the instantaneous voltage across the bolometer. Several $1 \times 1 \mathrm{~cm}$ bolometers with resistances between $25 \Omega$ and $75 \Omega$ were measured, and absolute responsivities between $0.75 \mathrm{mV} / \mathrm{W}$ and $1.0 \mathrm{mV} / \mathrm{W}$ were obtained, at a bias of $1 \mathrm{~V}$. and a modulation frequency of $100 \mathrm{~Hz}$. The discrepancy with the theoretical model arise from uncertainties in the values of $\alpha$ and $\mathrm{K}_{\mathrm{S}}$.

The absolute responsivity can also be found at $93 \mathrm{GHz}$, by measuring the source power with an accurate waveguide power meter, and using a calibrated transmitting horn antenna. This gives a plane-wave with a known absolute power density incident on the bolometer. The responsivity is then found from 
the measured voltage. It should be noted that in a practical setup, the bolometer is calibrated using a LF network, and the LF responsivity is used to measure the millimeter-wave power. However, by measuring the absolute responsivity at $93 \mathrm{GHz}$, one can determine the validity of the measured LF calibration. The absolute responsivities measured with a $93 \mathrm{GHz}$ system (and an $80.7 \mathrm{GHz}$ system) were within $10 \%$ of the LF responsivities. We attribute the discrepancies to uncertainties in our waveguide power meter, and to standing waves in the system. However, it is clear that the LF calibration gives accurate power measurements at millimeter and submillimeter wavelengths.

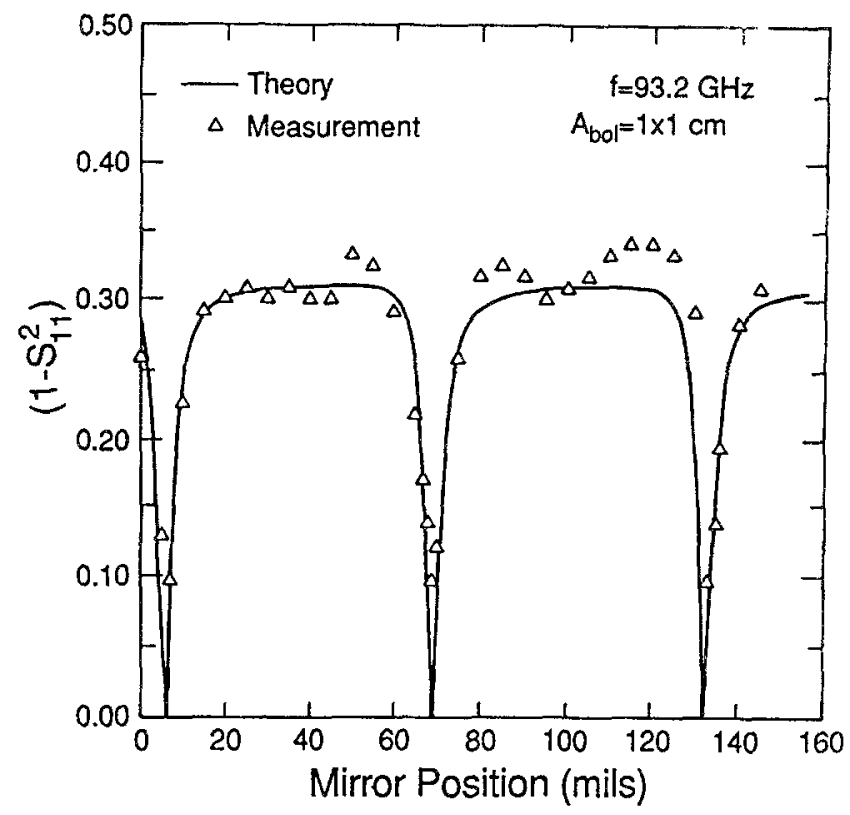

Figure 3. The computed and measured power absorbed by the bolometer vs. mirror position.

Finally, the noise of several $1 \times 1 \mathrm{~cm}$ bolometers was measured from $20 \mathrm{~Hz}$ till $100 \mathrm{KHz}$. The bolometer was not placed in a shielded box, but rather in a box with one side open to air. This resulted in some noise pick-up at low frequencies. The noise spectrum exhibits a $1 / \mathrm{f}$ rolloff till $1 \mathrm{KHz}$, and is limited by the Johnson noise for higher frequencies (Fig. 4). For a bias voltage greater than $2 \mathrm{~V}$., the bolometer heats up and the noise rises. The NEP is $3 \mu \mathrm{WHz}^{-1 / 2}$ at a video modulation of $1 \mathrm{KHz}$. 


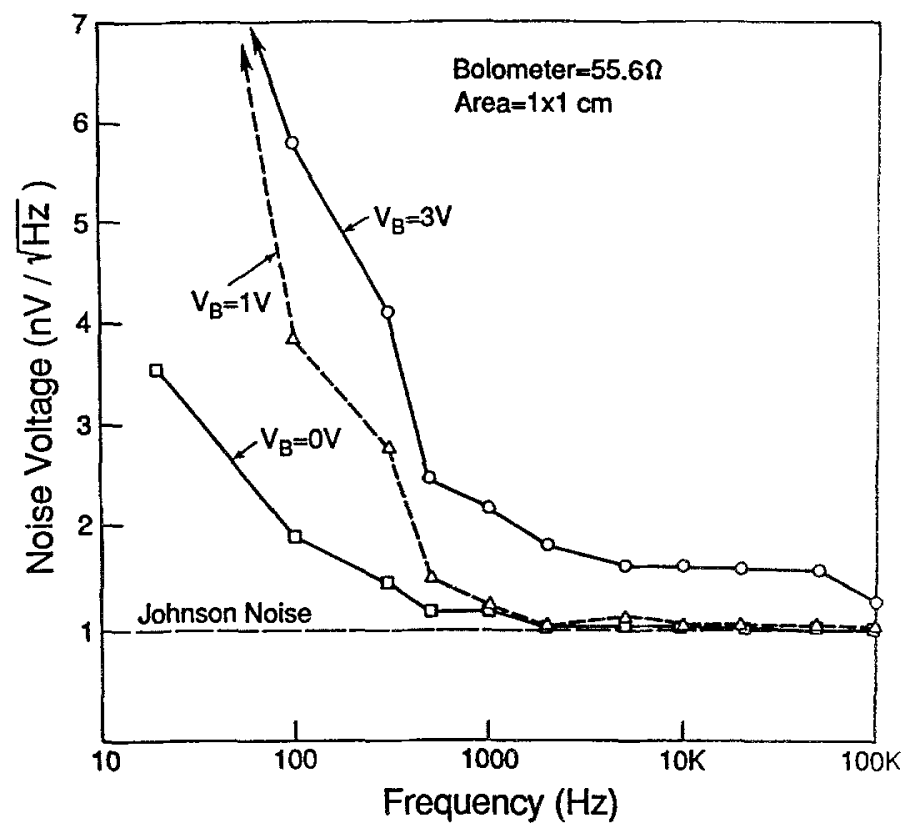

Figure 4. The measured noise-voltage of a large-area bolometer at different bias voltages.

\section{ACKNOWLEDGEMENTS}

The authors would like to thank Dr. Margaret Frerking and Dr. William McGrath for valuable discussions. This work was supported by the Innovative Space Technology Center at the Jet Propulsion Laboratory, and is now supported by the Center of Space Terahertz Technology at the University of Michigan, Ann Arbor.

\section{REFERENCES}

[1] D. P. Neikirk, W. W. Lam and D. B. Rutledge, " Far-infrared microbolometer detectors," Int. J. of Infrared and Millimeter Waves, vol. 5, No. 3, pp. 245-277, 1984.

[2] W. H. Block and O. L. Gaddy, "Thin metal-film room-temperature IR bolometers with nanosecond response times," IEEE Journal Quantum Electronics, vol. QE-9, No. 11, 1973.

[3] E. Holman, "Heat Transfer," First Edition, Mc GrawHill Book Company, New York, 1963. 